\title{
Applications of Aberration-corrected TEM and STEM in Complex Oxides and Nanostructured Materials
}

\author{
S. Lazar, ${ }^{* * *}$ L-Y. Chang, ${ }^{*}$ L. Gunawan, ${ }^{* * *}$, Y. Shao, ${ }^{* * *}$ and G. A. Botton**** \\ * Canadian Centre for Electron Microscopy, McMaster University, Hamilton Ontario, Canada \\ ** FEI Electron Optics, 5600 KA Eindhoven, The Netherlands \\ *** Department of Materials Science and Eng., McMaster University, Hamilton, ON, Canada
}

Aberration-corrected microscopy and new generations of instruments have the potential of significantly improving the development of new materials by providing superior imaging resolution, stability and spectroscopic capabilities. Applications of these techniques in the area of catalytic materials, functional oxides and electronic materials have emerged since the first aberrationcorrected microscopes were developed over 10 years ago [1-5]. New developments in commercial instruments have, furthermore, made it possible to significantly broaden the impact of these new technological developments. Here we report on example where aberration-corrected instruments have provided significant benefits in various areas of applications related to the study of electronic properties of complex oxides and nanoscale materials such as carbon nanotubes.

Experiments were carried out with a FEI Titan 80-300 Cubed equipped with two aberration correctors and a monochromator. This instrument achieved an information limit of $0.65-0.6 \AA$, a STEM information transfer of better than $0.7 \AA$ and an energy resolution in EELS of $0.13 \mathrm{eV}$.

STEM imaging of the multiferroic compound $\mathrm{Bi}_{2} \mathrm{Fe}_{\mathrm{x}} \mathrm{Cr}_{\mathrm{y}} \mathrm{O}_{6}$ has revealed the well-resolved transition metal sites between Bi bi-layers suggesting, directly from the High-Angle Annular Dark-Field HAADF images, similarities with an Aurivillius-type structure for this material (figure 1) that provides new insight on the magnetic properties of the thin films [6]. Energy-loss spectroscopy measurements at the $\mathrm{CrL}_{2-3}$ and $\mathrm{FeL}_{2-3}$ edges were also used to determine the valence of the $\mathrm{Fe}$ and $\mathrm{Cr}$ atoms. Phase contrast images also revealed additional information on the layer-by-layer growth mechanism of the films and the nature of defects at the interface with the $\mathrm{SrRuO}_{3}$ substrate.

With highly stable instrumentation, detailed information on the structure and roughness of interfaces can be provided by HAADF STEM. For example, in $\left(\mathrm{Ba}_{0.4} \mathrm{Sr}_{0.6}\right)\left(\mathrm{Ti}_{0.5} \mathrm{Nb}_{0.5}\right) \mathrm{O}_{3}$ metallic oxide thin films deposited on $\mathrm{MgAl}_{2} \mathrm{O}_{4}$ substrates, HAADF imaging (figure 2) provides direct evidence of lateral roughness at the level of few atomic cells. Combined with complementary focal series reconstruction of the phase contrast images, the STEM data provides detailed information necessary to understand the electronic structure changes of the first few atomic layers of these metallic oxide thin films in perfect epitaxial registry with the substrate (and less than $0.5 \%$ lattice mismatch). EELS data demonstrated the subtle changes in the $\mathrm{Ti} \mathrm{L}_{23}$ edges as compared to reference compounds with nominally similar $\mathrm{Ti}^{3+}$ valence (Figure 3 ). These results suggest that strain can induce valence changes.

Examples demonstrating the benefits of aberration-corrected low accelerating voltage microscopy in the study of carbon-based nanostructures show that it is possible to visualize single polymer chains wrapped around nanotubes (Figure 4) through the process of supramolecular functionalization [7] and other approaches[8,9]. 


\section{References}

[1] Haider, M., Rose, H., Uhlemann, S., Schwan, E., Kabius, B. and Urban, K. (1998) Ultram., Vol. 75, pp. 53-60.

[2] Haider, M., Rose, H., Uhlemann, S., Kabius, B. and Urban, K. (1998) J. of Electron Micr., Vol. 47, pp. 395-405.

[3] Haider, M., Uhlemann, S., Schwan, E., Rose, H., Kabius, B. and Urban, K. (1998) Nature, Vol. 392, pp. 768-769.

[4] Krivanek, O. L., Dellby, N. and Lupini, A. R., Ultramicroscopy, 78, (1999) 1-11.

[5] Krivanek, O. L., Nellist, P. D., Dellby, N., Murfitt, M. F. and Szilagyi, Z. Ultramicroscopy, 96, (2003) 229-237.

[6] R. Nechache, et al. J. Mater. Res. 22 (2007) p. 2102.

[7] F. Cheng et al., Macromolecules, 41, (2008) 9869-9874

[8] B. Bartova et al, Microscopy and Microanalysis 2009 (these proceedings)

[9] This research was supported by the Canada Foundation for Innovation, the Natural Sciences and Engineering council and the Ontario Centres of Excellence. We are grateful to A. Adronov, J. Preston, R. Hughes, Y. Zhao, A. Pignolet, R. Nechache, for providing several valuable samples and for valuable discussions.

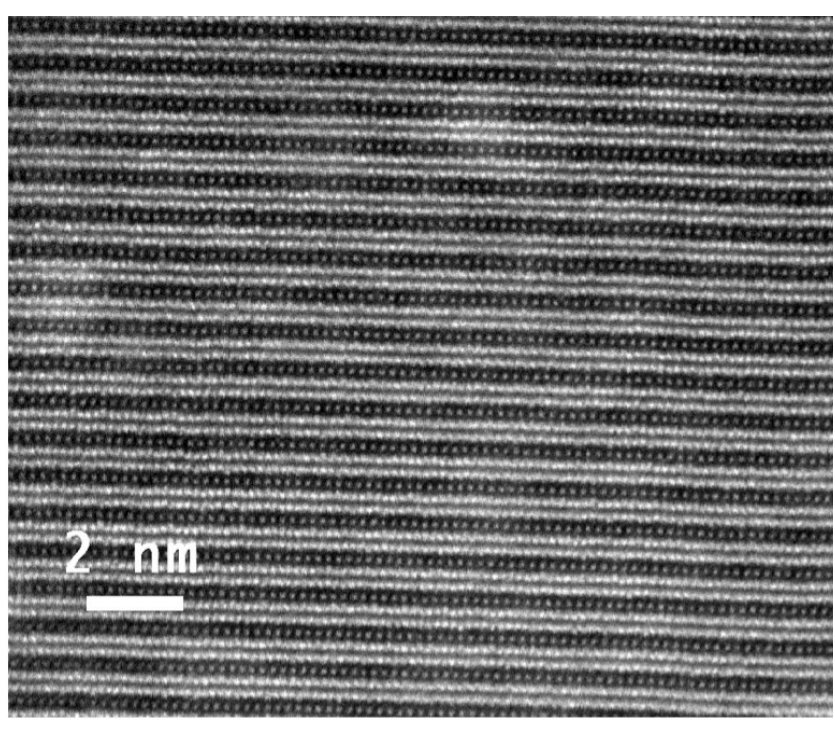

Figure 1: HAADF micrograph of $\mathrm{BiCrFeO}$ multiferroic material demonstrating the Bi-O bilayers and the transition metal perovskite slabs.

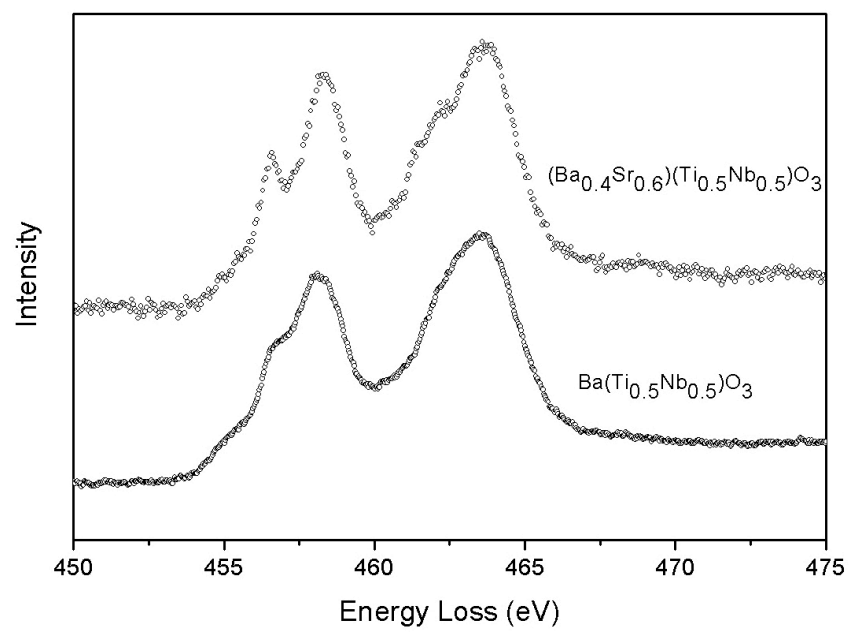

Figure 3 (above): $\mathrm{TiL}_{23}$ edges in $\left(\mathrm{Ba}_{0.4} \mathrm{Sr}_{0.6}\right)\left(\mathrm{Ti}_{0.5} \mathrm{Nb}_{0.5}\right) \mathrm{O}_{3}$ and $\mathrm{Ba}\left(\mathrm{Ti}_{0.5} \mathrm{Nb}_{0.5}\right) \mathrm{O}_{3}$.

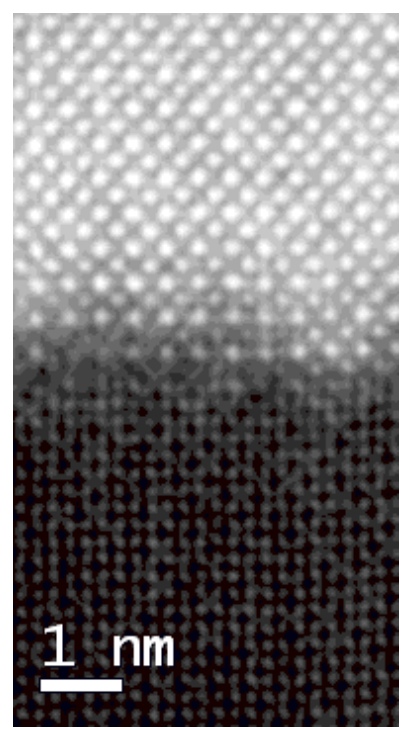

Figure 2: HAADF micrograph of the interface between (BaSr)(TiNb) $\mathrm{O}_{3}$ (top) and $\mathrm{MgAl}_{2} \mathrm{O}_{4}$ (bottom) demonstrating the interface roughness.

Figure 4 (right): Supramolecular functionalized single wall nanotube [7]

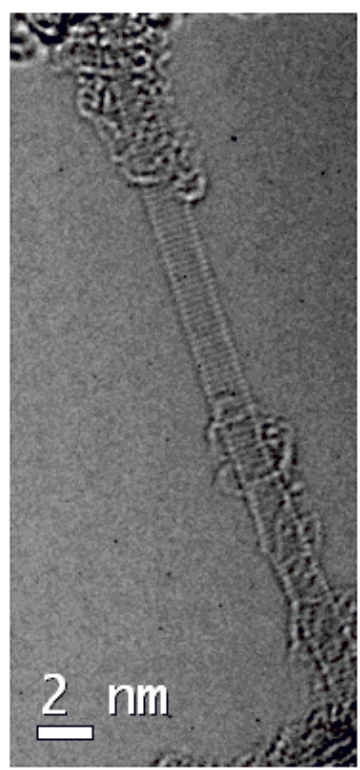

